\title{
Some Remarks on the Gribov Ambiguity
}

\author{
I. M. Singer
}

Department of Mathematics, University of California, Berkeley, California 94720, USA, and Massachusetts Institute of Technology, Cambridge, Massachusetts 02139, USA

\begin{abstract}
The set of all connections of a principal bundle over the 4-sphere with compact nonabelian Lie group under the action of the group of gauge transformations is studied. It is shown that no continuous choice of exactly one connection on each orbit can be made. Thus the Gribov ambiguity for the Coloumb gauge will occur in all other gauges. No gauge fixing is possible.
\end{abstract}

\section{$\S 1$. Introduction and Notation}

Let $\mathfrak{U}$ denote the set of all vector potentials (connections) for a fixed principal bundle $P$ with gauge group $G$ over a base space $M$. The group of the bundle $G$ is a compact nonabelian group and $M$ is an oriented Riemannian manifold. For each $A \in \mathfrak{A}$, let $F_{A}$ denote the field (curvature two form with values in the Lie algebra $g$ of $G$ ) of $A$ and $\left\|F_{A}\right\|^{2}$ the norm square or action. Let $(\mathfrak{B}$ denote the group of gauge transformations of $P$ (the automorphisms of $P$ which induce the identity map on $M)$. Since $(\mathfrak{H}$ is a group of transformations on $P$, it induces a group of transformations on $\mathfrak{A}$. If $\varphi \in \mathfrak{G}$ and $A \in \mathfrak{A}$, we denote this transformation by $\varphi \cdot A$. It is easy to see that $\varphi \cdot A=A-\left(D_{A} \varphi\right) \varphi^{-1}$ where $D_{A}$ is the covariant differential. In local coordinates, $D_{A}=\frac{\partial}{\partial x_{\gamma}}+A_{\gamma}$ so that $(\varphi \cdot A)_{\gamma}=-\frac{\partial \varphi}{\partial x_{\gamma}} \varphi^{-1}+\varphi A_{\gamma} \varphi^{-1}$. Also, $F_{\varphi \cdot A}=\varphi F_{A} \varphi^{-1}$ so that $\left\|F_{\varphi \cdot A}\right\|=\left\|F_{A}\right\|$.

Let $\mathfrak{N}=\mathfrak{U} / \mathfrak{G}$ the orbit space of $\mathfrak{U}$ under $\mathfrak{G}$, i.e., the set of equivalence classes where $A$ and $B$ are equivalent if there exists a $\varphi \in \mathbb{6 5}$ such that $B=\varphi \cdot A$. In the Feynman approach to quantum field theory one wants to make sense out of $\int_{\mathscr{U}} e^{-\left\|F_{A}\right\|^{2}}\{\} \mathscr{D} A / \int_{\mathfrak{I}} e^{-\left\|F_{A}\right\|^{2}} \mathscr{D} A$, where the integrand of the numerator may be constant on orbits of $\mathfrak{b}$. This introduces a difficulty because the orbits are expected to have infinite measure. One should really integrate over $\mathfrak{N}$, an intractable space. The physicists attempt to get round this difficulty by choosing a particular gauge, that is, choosing in a continuous manner one vector potential on each orbit; this choice is a map $s: \mathfrak{N} \rightarrow \mathfrak{U}$ such that $p \circ s=I$ where $p: \mathfrak{A} \rightarrow \mathfrak{N}$ is projection. They 
integrate over $s(\mathfrak{N})$ with a weight factor the Jacobian change of variables of $p: s(\mathfrak{N}) \rightarrow \mathfrak{N}$. The determinant weight factor is interpreted as the integral of a probability measure along the fibers (Fadeev-Popov ghosts).

To describe the particular gauge used, fix $A \in \mathfrak{A}$, and let $\mathscr{S}_{A}=\left[A+\tau ; D_{A}^{*} \tau=0\right.$, $\tau \in C^{\infty}\left(\Lambda^{1} \otimes g\right]$. Fixing $A$ makes the affine space $\mathfrak{A}$ a vector space isomorphic to $C^{\infty}\left(\Lambda^{1} \otimes g\right)$, equivariant 1 -forms on $B$ with values in $g$. The set $\mathscr{S}_{A}$ is easily seen to be the orthogonal compliment to the tangent space of the orbit of $\mathfrak{5}$ at $A$ for $D_{A}^{*}$ is the adjoint of the operator $D_{A}: C^{\infty}\left(\Lambda^{0} \otimes g\right) \rightarrow C^{\infty}\left(\Lambda^{1} \otimes g\right)$. When $M=\mathbb{R}^{4}, P$ the trivial bundle, and $A$ the 0 vector potential, then $\mathscr{S}_{A}=\left\{\tau_{\gamma} ; \sum_{\gamma=0}^{3} \frac{\partial \tau_{\gamma}}{\partial x_{\gamma}}=0\right\}$. In general, since $\mathscr{S}_{A}$ is perpendicular to the orbit at $A$, it does not intersect the orbit of $A$ near $A$, except at $A$. Generically, $A$ remains transversal to the orbits near $A$. One expected that $\mathscr{S}_{A}$ was a gauge: it intersected each orbit exactly once. We shall call $\mathscr{S}_{A}$ a generalized Coloumb gauge.

Recently Gribov [1] observed for $M=\mathbb{R}^{4}, P$ the trivial bundle, $G=\mathrm{SU}(2)$, $A=0$, and appropriate conditions at $\infty$, that the Coloumb gauge intersected $A$ at a large distance from $A$. He also discussed in detail the following situation. When $M=\mathbb{R}^{4}$, any $A$ is equivalent to a $B$ with $B_{0}=0$, where $B=B_{0} d t+\sum_{j=1}^{3} B_{j} d x_{j}$. Let $\mathfrak{A}_{0}=\left[A \in \mathfrak{A} ; A_{0}=0\right]$, the set of vector potentials for the trivial $\mathrm{SU}(2)$ bundle over $\mathbb{R}^{3}$ depending on a parameter $t$. The subgroup of $\left(\mathfrak{5}\right.$ leaving $\mathfrak{A}_{0}$ invariant as a set is the smooth functions of $\mathbb{R}^{3}$ into $\mathrm{SU}(2)$. With appropriate conditions at $\infty$, Gribov shows that the Coloumb gauge $\sum_{j=1}^{3} \frac{\partial A_{j}}{\partial x_{j}}=0$ intersects the orbit through the 0 vector potential at a large distance from 0 . More recently Jackiw et al. [2] have examined these ambiguities of the Coloumb gauge.

Since the Coloumb gauge is not a gauge, one naturally asks whether a true gauge exists. With no conditions at $\infty$, it is easy to exhibit some-algebraic gauges for example. When conditions are imposed at $\infty$, it is far from clear that gauges exist. The purpose of this note is to point out that if the conditions at $\infty$ amount to studying $M=S^{4}=R^{4} \cup \infty$ the unit sphere in $\mathbb{R}^{5}$ (or $M=S^{3}$ for any fixed time), then topological considerations imply that no gauge exists, i.e., no map $s$ exists. We also study the infinite dimensional bundles, connections, and curvature associated to $p: \mathfrak{U} \rightarrow \mathfrak{U} / \mathfrak{G}=\mathfrak{N}$. Though our results hold for any compact connected semi-simple group $G$, for purposes of clarity we restrict our attention to $G=\mathrm{SU}(N), N>1$, and we assume $M$ is compact. The condition at $\infty$ imposed by Gribov is that the gauge transformation from $\mathbb{R}^{3}$ (or $\mathbb{R}^{4}$ ) to $G$ extends to $S^{3}$ (or $S^{4}$ ) with $\varphi(\infty)=I$. The topological arguments will then still apply.

\section{§2. Results and Outline of Proofs}

Let $\mathfrak{b}_{A}=[\varphi \in \mathfrak{b} ; \varphi \cdot A=A]$, the isotropy or stability group at $A$. Since $\varphi \cdot A=A$ $-D_{A} \varphi \cdot \varphi^{-1}, \varphi \in \mathfrak{G}_{A}$ if and only if $D_{A} \varphi=0$. Hence such a $\varphi$ is determined by its value at a single point $m$ of $M$. If $E$ is the $N$-dimensional vector bundle over $M$ associated to the standard representation of $\mathrm{SU}(N)$ on $\mathbb{C}^{N}$, then $\mathfrak{G}$ is isomorphic to $C^{\infty}$ sections of $\operatorname{Hom}(E, E)$ which are special unitary at each point. Let $H_{A}$ denote the holonomy group of $A$ at $m$. Then $\varphi \in \mathfrak{W}_{A}$ if and only if $\varphi(m)$ commutes with $H_{A}$ 
and lies in $\mathrm{SU}(N)$. We call $A$ irreducible if $H_{A}$ is an irreducible subgroup of $\mathrm{SU}(N)$. When $A$ is irreducible, $\varphi \in \mathfrak{b}_{A}$ if and only if $\varphi(m)$ lies in the center of $\operatorname{SU}(N)$ $=\left\{e^{2 \pi i / N} I\right\}=Z_{N}$, the cyclic group of order $N$. Since $Z_{N}$ leaves every $A$ fixed, we introduce $\tilde{\mathfrak{G}}=\mathfrak{G} / Z_{N}$ which acts on $\mathfrak{A}$. Let $\mathscr{B}$ denote the set of irreducible connections in $\mathfrak{A}$, and let $\mathfrak{N}_{\mathscr{B}}=\mathscr{B} / \tilde{\mathfrak{G}}$. We remark that if $P$ is a nontrivial SU(2) bundle over $M$ and $H^{2}(M, Z)=0$, then $\mathscr{B}=\mathfrak{A}$. For a reducible vector potential $A$ would split $E$ into a direct sum of two line bundles trivial over $M$.

Theorem 1. $\mathscr{B}$ is an open dense set in $\mathfrak{A}$ and $\mathfrak{N}_{\mathscr{B}}$ is open and dense in $\mathfrak{N} . \mathscr{B}$ is a principal bundle over $\mathfrak{N}_{\mathscr{B}}$ with group $\tilde{\mathfrak{G}}$.

The topology of $\widetilde{\mathfrak{F}}$ is the $C^{\infty}$ relative topology as an open set in $\operatorname{Hom}(E, E)$. The $C^{\infty}$-topologies of the orbit spaces $\mathfrak{N}$ and $\mathfrak{N}_{\mathscr{B}}$ are the topologies as discussed in Ebin [3] and Palais [4]. They deal with the more difficult situation where $\mathfrak{A}$ is replaced by the space of metrics of $M$ and $\mathfrak{G}$ by $\operatorname{Diff}(M)$, the group of diffeomorphisms of $M$. The group $\operatorname{Diff}(M)$ is more complicated than the group of gauge transformations because the exponential map does not cover a neighborhood of the identity in $\operatorname{Diff}(M)$ whereas it does for $(\mathfrak{G}$. In short, the topology of $\mathfrak{N}$ is determined by the local slices $\mathscr{S}_{A} \cdot \mathfrak{N}$ is a metrizable Hausdorff space. $\mathfrak{N}_{\mathscr{R}}$ is an infinite dimensional $C^{\infty}$ Riemannian manifold whose tangent space at an orbit through $B$ is isomorphic to $\mathscr{S}_{B}$ and the inner product is given by $-\operatorname{tr}\left(\tau_{1} \wedge * \tau_{2}\right)$ where $B+\tau_{j} \in \mathscr{S}_{B}, j=1,2$.

Theorem 2. The homotopy groups $\pi_{j}(\mathscr{B})=0$.

The closed set $C=\mathfrak{A}-\mathscr{B}$ is a stratified set-the union of sub-manifolds of $\mathfrak{A}$. If $c \in C$, one can find an infinite dimensional subspace of the tangent space to $c$ in $\mathfrak{A}$ orthogonal to $C$ at $c$. As a result there is a neighborhood $\mathscr{U}$ of $c$ in $\mathfrak{A}$ such that $\pi_{i}(\mathscr{U}-\mathscr{U} \cap C)=0$. Suppose $f: S^{l} \rightarrow \mathscr{B} \subset \mathfrak{U}, S^{l}$ the boundary of an $l+1$ simplex $\Delta$. Since $\mathfrak{A}$ is affine, we can extend the map linearly to $\tilde{f}: \Delta \rightarrow \mathfrak{A}$, with the interior of the simplex possibly intersecting $C$. Use a small subdivision and the simplicial approximation theorem to find a map $\tilde{g}$ homotopic to $\tilde{f}$ so that the image of each small simplex under $\tilde{g}$ lies in some $\mathscr{U}$. Now move the $k$ dimensional skeleton off $C$ inductively beginning with $k=0$ and ending with $k=l+1$. The final map is homotopic to $\tilde{g}$ and lies in $\mathscr{B}$.

The set $C$ is a bifurcation set and has been analyzed for the group $\operatorname{Diff}(M)$ acting on the space of metrics by Fisher and Marsden [5] and Bourguignon [6]. The techniques used in the proof of Theorem 2 also show that the metrics with trivial isometry group is a classifying space for $\operatorname{Diff}(M)$.

If a continuous gauge $s: \mathfrak{R} \rightarrow \mathfrak{U}$ were to exist, then $\left.s\right|_{\mathfrak{R}_{\mathscr{B}}}: \mathfrak{N}_{\mathscr{B}} \rightarrow \mathscr{B}$ would give a cross section of the principal bundle $\mathscr{B}$, in which case $\mathscr{B}=\mathfrak{N}_{\mathscr{B}} \times \tilde{\mathfrak{G}}$. Since $\pi_{j}(B)=0$, we could conclude that $\pi_{j}(\tilde{\tilde{\mathfrak{G}}})=0$.

Theorem 3. $\pi_{j}(\tilde{\tilde{\mathfrak{F}}}) \neq 0$ for some $j$, for $M=S^{4}$ (or $\left.S^{3}\right)$.

Corollary 4. No continuous gauge $s: \mathfrak{R} \rightarrow \mathfrak{A}$ exists, for $M=S^{4}$ (or $S^{3}$ ).

To study the topology of $\tilde{\mathfrak{G}}$, we introduce some exact sequences. By definition, $0 \rightarrow Z_{N} \rightarrow\left(\mathfrak{G} \rightarrow \tilde{\mathfrak{G}} \rightarrow 0\right.$. Hence, if $\mathfrak{\mathfrak { F }}$ is connected, $\pi_{1}(\tilde{\mathfrak{G}}) \neq 0$; in general $\pi_{j}(\mathfrak{\mathfrak { G }}) \cong \pi_{j}(\tilde{\mathfrak{G}})$ for $j>1$ and $0 \rightarrow \pi_{1}(\mathfrak{\mathfrak { G }}) \rightarrow \pi_{1}(\tilde{\mathfrak{G}}) \rightarrow Z_{N} \rightarrow \pi_{0}(\mathfrak{\mathfrak { G }})$. To analyze $\mathfrak{\mathfrak { G }}$, let $\mathfrak{\mathfrak { G }}_{m}$ denote the gauge 
transformations that are the identity at $m$. We have $0 \rightarrow \mathfrak{5}_{m} \rightarrow \mathfrak{G} \rightarrow \mathrm{SU}(N) \rightarrow 0$. Hence $\pi_{j+1}(\mathrm{SU}(N)) \rightarrow \pi_{j}\left(\mathfrak{G}_{m}\right) \rightarrow \pi_{j}(\mathfrak{5}) \rightarrow \pi_{j}(\mathrm{SU}(N))$. In particular, since $\pi_{j}(\mathrm{SU}(N))=0$ for $j=1$ and $2, \pi_{l}\left(\mathfrak{G}_{m}\right) \cong \pi_{l}(\mathfrak{G})$ for $l=0$ and 1 .

Theorem 5. ${\left(\mathfrak{G}_{m}\right.}$ is weakly homotopic to the set of all maps $\{(M, m) \rightarrow(\mathrm{SU}(N), I)\}$ when $\operatorname{dim} M \leqq 4$. When $M=S^{r}, \pi_{j}\left(\mathfrak{G}_{m}\right) \cong \pi_{j+r}(\mathrm{SU}(N))$.

To see this, let $D^{ \pm}$denote the upper (lower) hemisphere of $S^{r}$ and $P^{ \pm}=\left.P\right|_{D^{ \pm}} \cong D^{ \pm} \times \mathrm{SU}(N)$. The principal bundle $P$ is determined by a patching map $\psi$ of the equator $S^{r-1} \rightarrow \mathrm{SU}(N)$. When $r=4$, the homotopy classes of such maps is determined by an integer $k$, the Pontrjagn index or minus the second Chern class of $P$. If $\varphi \in \mathbb{0}$, then $\varphi=\left\{\varphi^{+}, \varphi^{-}\right\}$where $\varphi^{ \pm}: D^{ \pm} \rightarrow \mathrm{SU}(N)$ and $\varphi^{-}=\psi \varphi^{+} \psi^{-1}$ on the equator $S^{r-1}$. Let $m$ be the north pole of $S^{r}$ so that $\varphi \in \mathfrak{G}_{m}$ implies $\varphi(m)=I$. Let $\beta: \varphi \rightarrow \varphi^{+}$mapping $\left(\mathfrak{G}_{m} \rightarrow\left[\varphi^{+}:\left(D^{+}, m\right) \rightarrow(\mathrm{SU}(N), I)\right]=\mathscr{H}\right.$. Clearly $\beta$ is a homomorphism with kernel $\mathscr{K}=\left[\varphi \in\left(\mathfrak{G}_{m} ; \varphi^{+}: D^{+} \rightarrow I\right]\right.$ which is the same as the maps $\left.\left.\varphi^{-}:\left(D^{-}, S^{r-1}\right) \rightarrow \mathrm{SU}(N), I\right)\right)$. Since $\mathscr{H}$ is contractible, the map $\varphi \rightarrow \varphi^{-}$of $\left(\mathfrak{G}_{m}\right.$ to $\mathscr{K}$ is a weak homotopy equivalence. Moreover $\left(D^{-}, S^{r-1}\right) \sim\left(S^{r}, n\right)$ so the first part of the Theorem holds for $S^{r}$. When $\operatorname{dim} M=4$, the same argument applies with $D^{+}$a ball about $m$ and $D^{-}=M-D^{+}$since $c_{1}=0$ and $c_{2}$ can be localized. When $\operatorname{dim} M<4, P$ is trivial so that $\mathfrak{F}_{m}=[\varphi:(M, m) \rightarrow(\mathrm{SU}(N), I)]$. Smoothing homotopies [7] and the fact that the smash product $S^{j} \wedge S^{r}=S^{j+r}$ gives the second statement of Theorem 5.

Theorem 3 and the subsequent Theorems 6 and 7 follow from Theorem 5, the known homotopy groups for $\mathrm{SU}(N)$, and the homotopy exact sequences for $0 \rightarrow Z_{N} \rightarrow \mathfrak{G} \rightarrow \tilde{\mathfrak{G}} \rightarrow 0,0 \rightarrow \mathfrak{G}_{m} \rightarrow \mathfrak{G} \rightarrow \mathrm{SU}(N) \rightarrow 0$. We provide some details using minimum information about $\pi_{j}(\mathrm{SU}(N))$ (see Toda [8]).

When $r=3, \quad \pi_{0}\left(\mathfrak{G}_{m}\right) \cong \pi_{3}(\mathrm{SU}(N)) \cong Z$. Since $\pi_{0}\left(\mathfrak{G}_{m}\right)=\pi_{0}(\mathfrak{G}) \quad$ and $\pi_{1}(\tilde{\mathfrak{G}}) \rightarrow Z_{n} \rightarrow \pi_{0}\left((\mathfrak{5})=Z, \pi_{1}(\tilde{\mathfrak{G}}) \neq 0\right.$ for $M=S^{3}$. When $M=S^{4}, \pi_{0}\left((\mathfrak{\xi})=\pi_{4}(\operatorname{SU}(N))=0\right.$ for $N>2$. Hence $\pi_{1}(\tilde{\mathfrak{G}}) \neq 0$ for $M=S^{4}$ and $G=\operatorname{SU}(N), N>2$. For SU(2), we noted that $\pi_{j}(\tilde{\mathfrak{5}})=\pi_{j}(\mathfrak{6})$ for $j=2$ or 3 while $\pi_{2}\left(\left(\mathfrak{b}_{m}\right) \cong \pi_{6}(\mathrm{SU}(2))=\pi_{6}\left(S^{3}\right)=Z_{12}\right.$. But $\pi_{3}(\mathfrak{5}) \rightarrow \pi_{3}(\mathrm{SU}(2)) \cong Z \rightarrow \pi_{2}\left(\mathfrak{5}_{m}\right) \cong Z_{12} \rightarrow \pi_{2}(\mathfrak{5}) \rightarrow \pi_{2}(\mathrm{SU}(2))=0$. Hence either $\pi_{2}(\mathfrak{5})$ or $\pi_{3}(\mathfrak{b})$ is not zero. This completes the proof of Theorem 3 .

Note that $\mathfrak{b}_{m}$ acts on $\mathfrak{U}$ without fixed points, for a gauge transformation leaving both a point of $P$ and a vector potential fixed is the identity. The last sentence of Theorem 5 implies that $\mathfrak{U} \rightarrow \mathfrak{U} / \mathfrak{G}_{m}$ has no cross section.

Theorem 6. For $M=S^{4}, \mathfrak{N}_{\mathscr{B}}$ is simply connected when $N>2$. For $\mathrm{SU}(2), \mathfrak{N}_{\mathscr{B}}$ is simply connected for Pontrjagn index $k$ odd and $\pi_{1}\left(\mathfrak{N}_{\mathscr{B}}\right)=Z_{2}$ for $k$ even.

Since $\mathscr{B}$ is homotopically trivial, $\pi_{1}\left(\mathfrak{N}_{\mathscr{B}}\right) \cong \pi_{0}(\tilde{\mathfrak{G}})$, which is 0 if $(\mathfrak{5}$ is connected. But $\pi_{0}(\mathfrak{5})=\pi_{0}\left(\mathfrak{G}_{m}\right)=0$ for $N>2$ as noted above. For $\mathrm{SU}(2)$, $\pi_{0}\left(\mathfrak{G}_{m}\right) \cong \pi_{4}(\mathrm{SU}(2)) \cong Z_{2}$. Thus $\pi_{0}(\tilde{\mathfrak{G}})$ is 0 if and only if $-I \in(\mathfrak{5}$ does not lie in the connected component of $\left(\mathfrak{G}\right.$ (from the sequence $0 \rightarrow Z_{2} \rightarrow(\mathfrak{G} \rightarrow \tilde{\mathfrak{G}} \rightarrow 0$ ). The map $-I$ can be connected to a map $\varphi \in \mathfrak{G}_{m}$, i.e. a map of $\left(S^{4}, m\right) \rightarrow\left(S^{3}=\mathrm{SU}(2), I\right)$. It turns out that this map is the suspension of a map $S^{3} \rightarrow S^{2}$ of index $k$. Hence $\varphi$ is homotopically trivial, i.e. lies in the component of the identity, if and only if $k$ is even.

Theorem 7. The principal bundle $\mathscr{B}$ with group $(\tilde{\mathfrak{b}}$ has no flat connection.

Since $\mathscr{B}$ is connected, so is $\mathfrak{N}_{\mathscr{B}}$. When $\mathfrak{N}_{\mathscr{B}}$ is simply connected, a flat connection would imply a continuous gauge. By Theorem 6 , we need only consider the case 
$N=2$ and $k$ even. Let $\tilde{\mathfrak{F}}_{0}$ be the component of the identity in $\tilde{\mathfrak{G}}$, so that $\mathscr{B} / \tilde{\mathfrak{F}}_{0}$ is the simply connected double cover of $\mathfrak{N}_{\mathscr{B}}$. A flat connection for $\mathscr{B}$ over $\mathfrak{N}_{\mathscr{B}}$ implies a cross section for $\mathscr{B}$ over $\mathscr{B} / \tilde{\mathfrak{G}}_{0}$ with group $\tilde{\mathfrak{G}}_{0}$. This does not exist because $\pi_{j}\left(\tilde{\mathfrak{G}}_{0}\right) \neq 0$ for some $j$. In fact $\pi_{j}\left(\tilde{\mathfrak{G}}_{0}\right)=\pi_{j}\left(\tilde{\mathfrak{F}}^{\mathbf{5}}\right)$ for $j>0$. But $\pi_{l}(\mathfrak{G}) \cong \pi_{l}\left(\mathfrak{G}_{m}\right) \cong \pi_{4+l}\left(S^{3}\right)=Z_{2}$ for $l=0,1$. From the sequence $0 \rightarrow \pi_{1}(\mathfrak{G}) \rightarrow \pi_{1}(\tilde{\mathfrak{G}}) \rightarrow Z_{2} \rightarrow \pi_{0}(\mathfrak{\mathfrak { G }}) \rightarrow \pi_{0}(\tilde{\mathfrak{G}})$, we can conclude that $\pi_{1}(\tilde{\mathfrak{G}}) \neq 0$.

Theorem 8. For every direction $A+s \mu \in \mathscr{S}_{A}$ there exists an $A+\tau \in \mathscr{S}_{A}$ such that $A+\tau$ is tangent to the orbit at $A+s_{0} \mu$.

Thus in every direction, the generalized Coloumb gauge eventually has a tangent to an orbit; this gives an infinity in the determinant of the Jacobian for the Fadeev-Popov approach. Theorem 8 is proved by noting that the tangent space to the orbit of $A+\mu$ is $\left[\left(D_{A}+\mu\right) f, f \in C^{\infty}\left(\Lambda^{0} \otimes g\right)\right]$. So we want to find an $s_{0} \ni \tau$ $=\left(D_{A}+s_{0} \mu\right) f$ and $D_{A}^{*} \tau=0$. Hence $\left(D_{A}^{*} D_{A}+s_{0} D_{A}^{*} \circ \mu\right) f$ must equal zero. Since the symbol of $D_{A}^{*} \circ \mu$ is not nonnegative, the self-adjoint operator $D_{A}^{*} \circ \mu$ is not nonnegative. Hence for sufficiently large $s_{0}, D_{A}^{*} D_{A}+D_{A}^{*} \circ s_{0} \mu$ has a null space. Set $\tau=\left(D_{A}+s_{0} \mu\right) f$ for $f \in \operatorname{ker} D_{A}^{*} D_{A}+s_{0} D_{A}^{*} \circ \mu, f \neq 0$.

Theorem 9. The set $\left[\tau ; A+\tau \in \mathscr{S}_{A}\right]$ is the horizontal space at $A$ of a connection of the bundle $\mathscr{B}$ with group $\tilde{\tilde{\mathfrak{G}}}$. The curvature of this connection is a 2-form which at $A$ assigns to the pair $\tau_{1}, \tau_{2}$ with $D_{A}^{*} \tau_{j}=0, j=1,2$ the Lie algebra value $\left(D_{A}^{*} D_{A}\right)^{-1}\left(b_{\tau_{1}}^{*}\left(\tau_{2}\right)\right.$ $-b_{\tau_{2}}^{*}\left(\tau_{1}\right)$, where $b_{\tau}$ is bracket by $\tau$.

Since $\tilde{\mathfrak{G}}$ acts as a group of isometries on $\mathfrak{A}$ and $\mathscr{B}, \tilde{\mathfrak{G}}$ preserves the orthogonal complement to the fiber. Hence we have a connection. Locally, $\mathscr{S}_{A}$ is a cross section, so gives a connection with 0 curvature. The curvature of the desired connection is computed by the usual formula for the difference of two curvatures in terms of the difference of the connections. In a subsequent paper we shall study the characteristic classes of $\mathfrak{N}_{\mathscr{B}}$ in terms of the curvature above as well as the characteristic classes of the tangent bundle of $\mathfrak{N}_{\mathscr{B}}$ in terms of the Riemannian curvature of $\mathfrak{N}_{\mathscr{B}}$ for the metric that $\mathscr{S}_{\mathscr{B}}$ defines on $\mathfrak{N}_{\mathscr{B}}$. Note that for $G$ abelian, brackets are 0 so that the adjoint of bracket, $b_{\tau}^{*}=0$. Hence the curvature is 0 and the connection is integrable. An integral submanifold provides the gauge for quantum electrodynamics.

Since $\mathfrak{N}_{\mathscr{B}}$ and $\mathfrak{N}_{\mathscr{B}}$ are paracompact, one way to avoid the difficulty posed by the Gribov ambiguity is to use a locally finite covering $\{\mathscr{V}\}$ and a subordinate partition of unity $\left\{f_{\mathscr{V}}\right\}$. For $\mathfrak{N}_{\mathscr{B}}$, the generalized Coloumb gauges $\mathscr{S}_{A}$ are cross sections locally. Use the Fadeev-Popov trick locally and weight by $f_{\mathscr{r}}$. On reducible connections $A \in \mathfrak{A}$, the $\mathscr{S}_{A}$ are only slices and intersect nearby orbits in a compact submanifold, an orbit of the stability group $\mathfrak{G}_{A}$. This is not a serious difficulty for one can still find a local cross section. This partition of unity argument would be useful if the covering used could be made explicit.

Acknowledgements. We thank M. F. Atiyah, J. Glimm, R. Palais, R. Stora, and E. Spanier for fruitful discussions. This research was supported by the National Science Foundation under Grant MCS 74$05913 \mathrm{~A} 05$. 


\section{References}

1. Gribov,V.N.: Instability of non-abelian gauge theories and impossibility of choice of Coloumb gauge. SLAC Translation 176 (1977)

2. Jackiw, R., Muzinick, I., Rebbi, C.: Coloumb gauge description of large Yang-Mills field. Phys. Rev. 17, $1576(1978)$

3. Ebin,D.: The manifold of Riemannian metrics. In: Proceedings of symposia in pure mathematics, Vol. 15, pp. 11-40. Providence, Rhode Island: American Mathematical Society 1970

4. Palais, R.: Foundations of global non-linear analysis. New York: W. A. Benjamin, Inc. 1968

5. Fischer, A., Marsden, J. : The space of conformally related metrics. Can. J. Math. 29, 193-209 (1977)

6. Bourguignon, J.: Une stratification de l'espace des structures riemanniennes. Compositio Math. 30, $1-42(1975)$

7. Munkries,J.R.: Elementary differential topology. In: Annals of mathematics studies, Vol. 54. Princeton, New Jersey: Princeton University Press 1963

8. Toda,H.: Composition methods in homotopy groups of spheres. In: Annals of mathematics studies, Vol. 49. Princeton, New Jersey: Princeton University Press 1962

Communicated by J. Glimm

Received January 30, 1978 